$4 \frac{18}{10}-28-91 \% 5$ PREPARED FOR THE U.S. DEPARTMENT OF ENERGY, UNDER CONTRACT DE-AC02-76-CHO-3073

PPPL-2792

PPPL-2792

UC-427

\title{
ANGULAR MOMENTUM, g-VALUE, \\ AND MAGNETIC FLUX OF GYRATION STATES
}

BY

V. ARUNASALAM

October 1991

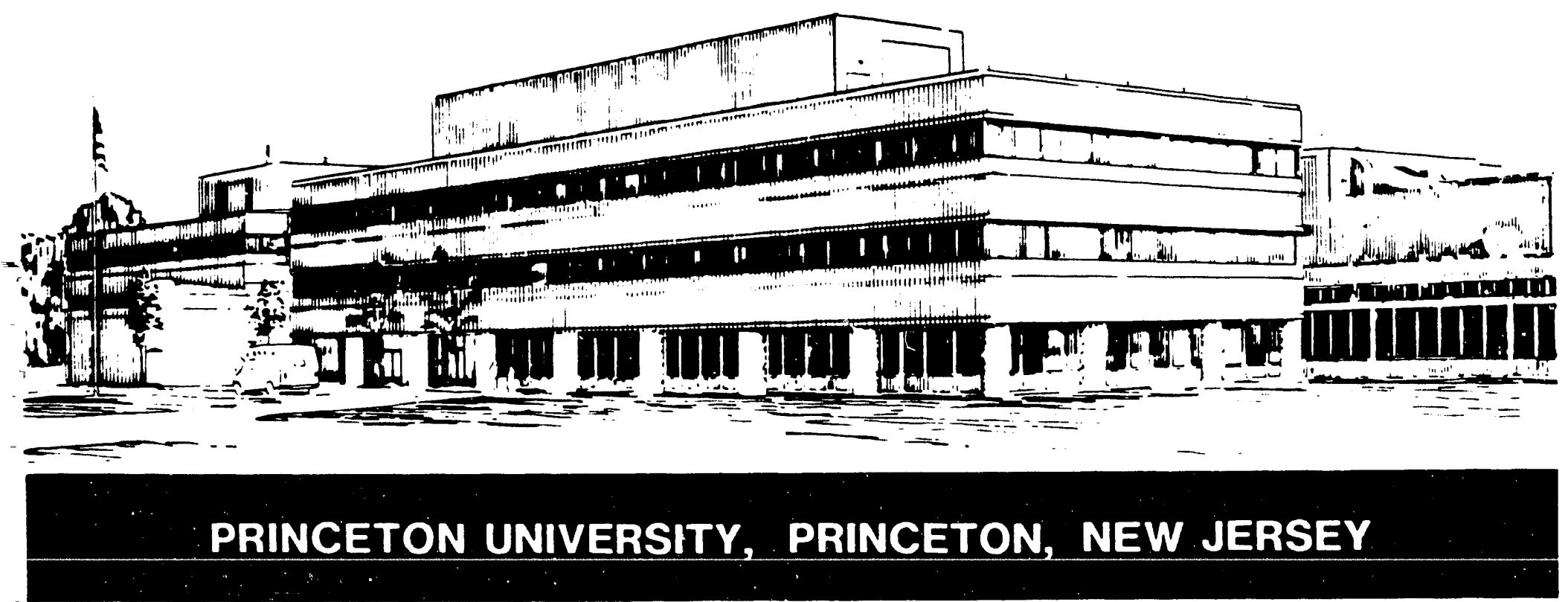




\section{NOTICE}

This report was prepared as an account of work sponsored by an agency of the United States Government. Neither the United States Government nor any agency thereof, nor any of their employees, makes any warranty, express or implied, or assumes any legal liability or responsibility for the accuracy, completeness, or usefulness of any information, apparatus, product, or process disclosed, or represents that its use would not infringe privately owned rights. Reference herein to any specific commercial produce, process, or service by trade name, trademark, manufacturer, or otherwise, does not necessarily constitute or imply its endorsement, recommendation, or favoring by the United States Government or any agency thereof. The views and opinions of authors expressed herein do not necessarily state or reflect those of the United States Government or any agency thereof.

\section{NOTICE}

This report has been reproduced directly from the best available copy.

Available to DOE and DOE contractors from the:

Office of Scientific and Technical Information

P.O. Box 62

Oak Ridge, TN 37831 ;

Prices available from (615) 576-8401.

Available to the public from the:

National Technical Information Service

U.S. Department of Commerce

5285 Port Royal Road

Springfield, Virginia 22161

703-487-4650 
ANGULAR MOMENTUM, g-VALUE, AND MAGNETIC FLUX OF GYRATION STATES

\author{
V. Arunasalam \\ Princeton University. \\ Princeton Plasma Physics Laboratory. \\ Princeton, New Jersey, 08543
}

PPPL--2792

DE92 001932

ABSTRACT

Two of the world's leading (Nobel laureate) physicists disagree on the definition of the orbital angular momentum $L$ of the Landau gyration states of a spinless charged particle in a uniform external magnetic field $B=B i_{z}$. According to Richard $P$. Feynman (and also Frank Wilczek) $L=(r \times \mu v)=r \times(p-$ QA/C). while Felix Bloch (and also Kerson Huang) defines it as $L=$ rxp. We show here that Bloch's definition is the correct one since it satisfies the necessary and sufficient condition $L x L=$ in $L$, while Feynman's definition does not. However, as a consequence of the quantized Aharonov-Bohm magnetic flux, this canonical orbital angular momentum (surprisingly enough) takes half-odd-integral values with a zero-point gyration state of $L_{z}=\uparrow_{1} / 2$. Further, since the diamagnetic and the paramagnetic contributions to the magnetic moment are interdependent, the g-value of these gyration states is two and not one, again a surprising result for a spinless case. The differences between the gauge invariance in classical and quantum mechanics, Onsager's suggestion that the flux quantization might be an intrinsic property of the electromagnetic field-charged particle interaction, the possibility that the experimentally measured fundamental unit of the flux quantum need not necessarily imply the existence of "electron pairing" of the Bardeen-Cooper-Schrieffer superconductivity theory, and the relationship to the Dirac's angular momentum quantization condition for the magnetic monopole-charged particle composites (i.e., Schwinger's dyons), are alsc briefly examined from a pedestrian viewpoint.

\title{
MASTER
}




\section{INTRODUCTION}

In classical mechanics.' it is well known that in the Lagrangian formalism the fundamental variables are the generalized coordinates $r$ or $x_{j}$ and the corresponding velocities $v=\dot{r}=d r / d t$ or $v_{j}=\dot{x}_{j}=d x_{j} / d t$ and the Lagrangian $\mathcal{L}(r, v, t)=T(v, t)-U(r, v, t)$, where $T$ is the kinetic energy and $U$ is the "generalized potential". For a spinless charged particle of charge $q$ in an electromagnetic field given by the scalar potential $\Phi$ and the vector potential $A$, this generalized potential is velocity dependent and is given by $U=q \Phi$ $q A \cdot v / c$. However, in the Hamiltonian rormalism the independent variables are the generalized coordinates $r$ and the corresponding generalized canonical momenta $P$ defined as $P=\partial \mathcal{L}(r, v, t) / \partial v$. Thus, for the charged particle of mass $\mu$ in this electromagnetic field, $P=\mu v+q A / c$. That is, the "canonical momentum," or the "dynamical momentum" or the " $P$-momentum" 2 is the sum of the "mechanical" or the "kinetic" or the " $\mu v-m o m e n t u m " 3 \mu v$ and the "potential momentum" or the "Aharonov-Bohm momentum" QA/c. In quantum mechanics, which is based on the Hamiltonian formalism, 4.5 the operator $p$ is the displacement operator $-i \hbar \nabla=-i n a / \partial r$, and the free particle wave function $\psi$ is proportional to $\exp (i p \cdot r / h)$. That is, for any arbitrary functior, $f(r)$, one can show that. $f(r+a)=\Sigma_{n}\left[(a \cdot \nabla)^{n} / n !\right] f(r)=[\exp (a \cdot \nabla)] f(r)=[\exp (i a \cdot p / \pi)] f(r)$. Thus, the Aharonov-Bohm phase 2.6 due to the Aharonov-Bohm momentum is $\exp (\mid Q A \cdot r / T C)$.

Since for the charged particle in the electromagnetic field $P=\mu v$. $q A / C, \quad r \times p=r \times \mu v+q r \times A / C$. ODviously, $\quad r \times A / C$ represents the argular momentum contribution from the charged particle-electromagnetic field interaction. It should be noted that this field contribution to the angular momentum depends only on the finite value of the vector potential $A$ and nct on the magnetic rield B. For example, just outside an infinitely long solencid 
$B=0$, but $A$ is nonzero, and hence this field contribution to the angular momentum is finite. Note that this is a field-particle interaction angular momentum, and is not the usual electromagnetic field angular momentum which is given by the volume integral ${ }^{7.8} \mathrm{~K}=(1 / 4 \pi c) \int d V \mathrm{r} \times\left(E^{*} \times H+E \times H^{*}\right)$. The question then arises as to what is the orbital angular momentum of the particle?. Is it $\operatorname{rxp}$ or is it rxuv?. In the literature two of the world's leading (Nobel laureate) physicists disagree on the definition of this orbital angular momentum $L$ of the farticle. According to Richard P. Feynman ${ }^{9}$ (and also Frank wilczek, 10 and possibly one group of physicists) $L=(r \times \mu v)=r \times(p-$ $Q A / C$ ), while Felix Bloch 11 (and also Kerson Huang, ${ }^{12}$ and possibly another group of physicists) defines it as $L=r \times p$.

It is our aim in this paper to show that Bloch's definition is the correct one since it satisfies the necessary and sufficient condition $L X L=i \hbar L$, (i.e., the commutation relations $\left[L_{j}, L_{k}\right]=i \hbar L_{1}, j, k, 1$, in cyclic order); while Feynman's definition does not. We will then show that for the the Landau cyclotron motion about its guiding center, 13 as a consequence of the quantized Aharonov-Bohm magnetic flux, this canonical orbital angular momentum, surprisingly enough, takes half-odd-integral values, with a zero-point gyration state of $L_{z}=\pi / 2$. This of course implies that this particular problem with the vector potential expressed in the cylindrically symmetric gauge ( i.e., $A=$ Bxr/2) does not have an exact Schrodinger's spatial represeriation. Then we examine the striking similarity between this zero-point Landau gyration state and the Dirac's free particle Zitterbewegung motion.14,15,16 Further, since the diamagnetic and paramagntic contributions to the magretic moment are irterdeperdent, we find that the canonical g-value or these gyration states is two and not ore, again a surprising result for a spirless particle case. cf course, the $g$ value of two is entirely consistent with the half-irtegral $L_{z}$-values. We show that $g=2$ both classically and then quantum 
mechanically. Indeed, the rigorous solutions of the Landau gyration states using the equations of motion in the Heisenberg's matrix representation 17 agree exactly with Huang's solutions 12 using the "old" quantum theory via the quantization of the action integral $S$ using the Bohr-sommerfeld-Wilson (BSW) quantization condition. 3 We then discuss the differences between the gauge invariance of the classical dynamical variables, and the gauge invariance of the corresponding quantum mechanical Hermitian operators. Indeed, a gauge invariant dynamical variable in classical mechanics goes over as the corresponding gauge-dependent Hermitian operator. We then examine the flux quantization and the fundamental unit of magnetic flux using a specific model problem of a solid cylinder of free electron gas in a uniform external magnetic field. We will show that even though the flux $\Phi_{j}=(h c / q)(j+1 / 2)$ enclosed Dy the successive Landau orbits steps in units of $\mathrm{hc} / \mathrm{q}$, at sufficiently low temperatures, the average flux $\left\langle\Phi_{j}\right\rangle=(h c / 2 q)(j+i)$ is quantized in units of the fundamental unit of flux hc/2q, which is characteristic of the zero-point gyration ground state. This may indicate the possibility that the experimentally observedi8 fundamental unit of the flux quantum as $\mathrm{hc} / 2 \mathrm{a}$ instead of as hc/a need not necessarily imply the existence of "electron pairs" of the Bardeen-Cooper-Schrieffer (BCS) theory of superconductivity. 19 a rather controversial. speculative and outrageous result. Since flux quantization is a consequence of the angular momentum quantization, there are indications that this might be an intrinsic property of the electromagnetic field-charged particle interaction, in agreement with onsager's suggestion.20,21 finally, we give a brief discussion of schwinger's charge-monopole composites or dyors, 22,23.24 and Wilczek's charged particle-flux tude composites. 10 Here, we find that the velocity-dependent particle-electromagnetic field interacticr contribution to the total angular momentum plays the role of a spin argular momentum. possibly implying the usual connection between spir and statistirs as discussed by Goldhaber. 25 
11. PRELIMINARIES

We now consider the properties of the angular momentum operator $J$. We work entirely with a representation at a particular instant of time, so that we are not concerned here with the change in time of the resulting angular momentum matrices. However, if the angular momentum commutes with the Hamiltonian, it is a constant of motion, and the matrices retain their form for all time. In the Hamiltonian quantum mechanics, following Schiff, 4 we define the angular momentum $J$ of a particle about some point in terms of its displacement $r$ from that point and its canonical momentum $p$ as

$J=r \times p$.

We do not require that $P$ be expressible as differential operator, but rather that the components of $r$ and $p$ satisfy the fundamental commutation. reiations

$\left[r_{j}, P_{k}\right]=$ it $\delta_{j k} ;\left[r_{j}, r_{k}\right]=\left[p_{j}, P_{k}\right]=0$

where $\delta_{j k}$ has the usual meaning of the kronecker $\delta$ symbol; j, $k=1,2$, and 3: $r_{1}=x, r_{2}=y, r_{3}=z$; and $p_{1}=p_{x} \cdot p_{2}=p_{y}$, and $p_{3}=p_{z}$. It is then possible to find commutation relations between the components of $J$ that do not involve $r$ and $p$ as

$J \times J=$ inj or $\left.\left[j_{j}, j_{k}\right]=i \hbar\right\lrcorner_{1} ; j, k, 1$, in cyclic order.

Now it turns out that the relations of Eq.(3) admit of scme ratrix representation for $J$ that are not compatible with the original definition of Eq.(1). Specifically, in the literature it is stated as a theorem $4,5,13$ that the 
matrices for the components of the orbital angular momentum of a particle $L$ $=J$ of Eqs.(1) and (2) with $P=-i t \nabla$ in the schrodinger's spatial representation must have eigenvalues that are integer multiples of $h$, and they cannot have eigenvalues that are half-odd-integer multiples of $h_{i}$ while the matrices for the components of $J$ of Eq.(3) can have eigenvalues that are either integer or half-odd-integer multiples of $\AA$. This theorem for the orbital angular momentum is based on the fact that the particle wave function for continuity be single valued for a rotation about any axis through an angle $2 \pi$, and the angular momentum eigenfunctions are the ordinary spherical harmonics. However, in the study of rigid body rotations in terms of Cayley-Klein parameters, ${ }^{1}$ one encounters a double-valued isomorphism between a set of three-by-three real orthogonal matrices (appropriate to rotations in the physical three-dimensional space) and the corresponding set of pairs of two-by-two complex unitary matrics (appropriate to rotations in the complex two-dimensional spinor space). The two complex unitary matrices that form the pair are such that one is the negative of the other. Indeed, in this spinor space with the Pauli spin matrices as the unit rotators, the occurrence of half angles and the resultant double-valued property are intimately connected with the fact that the spin is half integral. Although the wave runction may de double valued under rotation, all physically observable properties remain single valued, of course. This is the reason that Eq.(3) admits some matrix representations for $J$ that are not compatible with the original definition of Eq.(1). When such a conflict arises, there is in some cases physical reason to regard EQ.(3) as more funoamental than Eq.(1).

The angular momentum $J$ can also be defined in terms of infinitesimal rctation $R$ that permits of generalization to more complicated systems (many interacting particles, spins, fields). The effect of a rotation about the $z$-axis through an infinitesimal angle $\delta \varphi$ on an arbitrary function $f(x, y, z)$ is 
$R_{z}(\delta \varphi) f(x, y, z)=[1+\delta \varphi((y \partial / \partial x)-(x \partial / \partial y)\}] f(x, y, z)=\left[1+(\delta \varphi / i \hbar) J_{z}\right] f(x, y, z) \cdot(4)$

Here, $J$ is given by Eq.(1) but now with $P$ expressible as the differential operator $-i \hbar \nabla=-i \hbar \partial / \partial r$. Since Eq.(4) is true for all arbitrary functions $f(x, y, z)$, we can write the z-axis infinitesimal rotation operator as

$R_{Z}(\delta \varphi)=1+(\delta \varphi / i \hbar) J_{Z}, \quad \delta \varphi$ infinitesimal

From Eq.(5), one can show that for rotations about the z-axis through a finite angle $\varphi, R_{Z}(\varphi)=\exp \left[(i \varphi / \hbar) J_{Z}\right]$, since as $n \rightarrow \infty,(1+a / n)^{n} \rightarrow \exp (a)$, where $\delta \varphi$ $\varphi / n$ and $a=(i \varphi / h) J_{Z}$. Equation (5) is as equally fundamental as Eq.(3), and can be used to define $J$ even when the $r$ and $p$ that appear in Eq.(1) do not exist. Thus, any angular momentum operator $J$ must satisfy the commutation relations of Eq.(3), and this in turn will satisfy the infinitesimal rotation requirements of Eq.(5). However, it is important to realize that in Eq.(1) we do require that the components of $r$ and $p$ satisfy the fundamental commutation relation of Eq.(2), if $J$ is to be an angular momentum operator. 4,15 Then and only then will the components of $J$ satisfy the recessary and sufficient condition of Eq.(3).

Armed with this knowledge, we are now in a position to examine the correctness of some of the definitions of the angular momentum found in the literature. ${ }^{4,9-12}$ it is clear that if the generalized potential $U$ of the Lagrangian of the problem under study does not depend on the particle velocity. then there is no potential momentum and the canonical momentum $p$ is identical to the mechanical momentum $\mu v$. Then, there is no ambiguity in the definition of the orbital angular momentum as $J=L=r \times p=r \times \mu v$. However. when $U$ is velocity dependent, $P=\mu v$. Then, what is the orbital angular momentum $L ?$. Is it $r \times p$ or $r \times \mu v$ ?. Specifically. what is the orbital 
angular momentum $L$ of a spinless charged particle of charge $q$ and mass $\mu$ in an electromagnetic field of vector potential A?. In the literature, two of the world's leading (Nobel laureate) physicists disagree on the definition of the orbital angular momentum $L$ of the gyration states of a spinless charged particle in a uniform external magnetic field $B=B i_{Z}$, whose vector potential is conveniently taken here in the cylindrically symmetric form $A=B \times r / 2$. According to Richard $P$. Feynman ${ }^{9}$ (and also Frank wilczek $\left.{ }^{10}\right) L=r \times \mu v=r x(P$ - $q A / C$ ), while Felix Bloch 11 (and also Kerson Huang ${ }^{12}$ ) defines it as $L=\operatorname{rxp}$. Feynman states that "From analogy to classical physics, one might expect that the angular momentum operator is now $L=r x(p-q A / c)$." $B y$ using this definition for the orbital angular momentum $L$ he goes on to show from the Dirac's relativistic Hamiltonian that $\mathrm{dL} / d t=\dot{L}=0$ even with central forces. Then he shows that $d[L+\hbar \sigma / 2] / d t=0$ for central forces, where $\sigma$ is the familiar Pauli spin matrix. Thus, he states that "The operator $L+\hbar \sigma / 2$ may De regarded as the total angular momentum operator. where $L$ represents orbital angular momentum and ho/2 intrinsic angular momentum for spin $1 / 2$. Thus total angular momentum is conserved with central forces." In order to discuss this same problem. schiff ${ }^{4}$ on the other hand plays it very safely by assuming at the start itself that $A=0$ and defines this orbital angular momentum as $L=r \times p$, and shows that $d L / d t=-d\left(\pi \sigma^{\prime} / 2\right) / d t=0$ both for the central forces and also for a completely free Dirac particle. This nonphysical behavior of the Dirac free particle is a direct consequence of the lack of manifest Lorentz covariance of the Dirac free particle Hamiltonian as discussed by this author in earlier publications. 15.26 Frank wilczek 10 states that "It is demonstrated that the orbital angular momentum $L_{z}$ of a particle of charge a crbiting around a tube with magnetic flux $\Phi$ is quartized ir units $L_{z}=$ integer - $q \Phi / 2 \pi$." This is an interesting result and we w/ll ccme back to it later. A somewhat related problem of the possibllity of half-odd-integral crbital angular momentum has been discussad by this author 27 
in an unpublished Princeton University Plasma Physics Laboratory Report (MATT-755) in February. 1970. As one method of proof wilczek states "Rotations around the z-axis, i.e., changes in the azimuthal angle $\varphi$, are generated by the covariant angular momentum $L_{z}=-i \partial_{\varphi}-q A_{\varphi}$. In a nonsingular gauge, $A_{\varphi}=\Phi / 2 \pi$ and the azimuthal dependence of the electron wave function is $\psi_{n} \propto \exp (i n \varphi), n=$ integer for continuity. Then $L_{z} \psi_{n}=(n-$ $q \Phi / 2 \pi) \Psi_{n}$, in agreement with the previous conclusion." It is clear that according to wilczek the orbital angular momentum component $L_{z}$ defined in terms of infinitesimal rotations about the $z$-axis is given by $(i z \cdot L)=$ $\left[i_{z} \cdot(r \times \mu v)\right]=\left[i_{z} \cdot\{r \times(p-q A / c)\}\right]=-i \hbar \partial / \partial \varphi-q A_{\varphi} / c$. Here, by covariant angular momentum wilczek means the yauge covariance and not the relativistic Lorentz covariance. Wilczek examines the problem of a particle of charge a which orbits around, but does not penerate, a solenoid running along the z-axis. Here, at the location of the particle there is no $B$-field, but there is an $A$. The entire $B$-field exists only inside the solenoid. This is not a steady state problem, but a transient one. Before the current is turned on in the solenoid, the particle experiences no force and hence moves in a straight line and cannot orbit around the solenoid. If a current is slowly turned on, the charged particle will reel an electric field $E(r)=-\left(i_{z} \times r_{\perp}\right)\left(\dot{\Phi} / 2 \pi c r_{\perp}^{2}\right)$ according to Faraday's law, where $\Phi$ is the flux through the solenoid. This gives the transient change in the angular momentum $\dot{L}_{z}=\left[r_{\perp} \times(q E)\right]_{z}=(q \Phi / 2 \pi c)$. However, once the current in the solenoid has reached its steady state value, $\dot{\Phi}$ $=C, E=0$, anc $\dot{L}_{z}=0$, and the particle again moves in the force free straight line motion and cannot oroit around the sol moid. Thus, in the transient stage, the angular momentum is quantized in units $L_{z}=$ integer $-q \Phi / 2 \pi c$. This is nowever not true in the steady state where $\Phi$ is zero, while $\Phi$ may be nonzero. Let us now apply wilczek analysis to the classical cyclotron moticr. 
about the guiding center of a spinless charged particle in this uniform magnetic field $B=B i z$, whose vector potential in the cylindrically symmetric gauge is $A=B \times r / 2=(B / 2) i_{z} \times r_{\perp}$. Here, $\perp$ refers to directions perpendicular to the B-field. In sharp contrast to Wilczek's problem, this is a steady state and not a transient problem. Further, at the location of the particle, both $B$ and $A$ are nonzero and are related as $B=\nabla \times A$.

$L_{Z} i_{Z}=r_{\perp} \times \mu v_{\perp}=r_{\perp} \times p_{\perp}-(q B / 2 C)\left(r_{\perp} \times i_{Z} \times r_{\perp}\right)=r_{\perp} \times P_{\perp}-(q B / 2 c)\left(r_{\perp} \cdot r_{\perp}\right) i_{Z}$

$$
=r_{\perp} \times p_{\perp}-(q / 2 \pi c)\left(\pi r_{\perp}^{2} B\right) i_{Z}=\left[-i \hbar \partial / \partial \varphi-q A_{\varphi} / c\right] i_{Z} \text {. }
$$

where $A_{\varphi}=\left(\pi r_{\perp}^{2} B\right) / 2 \pi=\Phi / 2 \pi, \Phi=\pi r_{\perp}^{2} B$ is the magnetic flux enclosed by the cyclotron orbit of radius $r_{\perp}, P=-i \hbar \nabla$, and we have used the relation $\left(r_{1} \times i_{Z} \times r_{1}\right)=\left(r_{1} \cdot r_{1}\right) i_{Z}-\left(r_{1} \cdot i_{Z}\right) r_{1}=\left(r_{1} \cdot r_{1}\right) i_{Z}$, since $r_{\perp} \cdot i_{Z}=0$. If we now assume that the azimuthal dependence of the particle wave function is $\Psi_{m} \propto$ $\exp (i n \varphi \varphi), \quad m=$ integer for continuity and single valuedness for rotations through $2 \pi$, then Eq.(6) yield the Wilczek's result $L_{z}=$ [integer]th $-q \Phi / 2 \pi c$.

Felix Bloch, 11 on the other hand, defines this orbital angular momentum of such a particle in the vector potential $A$ as $L=r \times p$, and he assumes that the components of this orbital angular momentum must have eigenvalues that are integer multiples of $\boldsymbol{h}$. In this way he tries to interpret some of the characteristic features of superconductivity in terms of general principles cf physics. Kerson Huang, 12 in his discussion of the Landau diamagnetism (at high encugh temperatures) and the De Hass-van Alphen effect (at low encugh temperatures), also defines this orbital angular momentum as $L=r \times p$, ir agreement with Bloch and Schiff but in disagreement with Feynmar and wilczek. 
However, Huang assumes that the components of this orbitil angular momentum must have eigenvalues that are half-odd-integer multiples of $\hbar$, in disagreement with Bloch. He makes use of the "old" quantum theory and assumes that the action integral $S$ over one complete cycle of motion is

$$
\begin{aligned}
S & =\oint p_{\perp} \cdot d r_{\perp}=\oint p_{\perp} \cdot(r \times d \Omega)_{\perp}=-\int\left(r_{\perp} \times p_{\perp}\right) \cdot d \Omega_{Z} i_{Z}=-2 \pi L_{Z} \\
& =(n+1 / 2) 2 \pi n=(n+1 / 2) h \quad(n=0,1,2, \ldots),
\end{aligned}
$$

where $d \Omega$ is the infinitesimal rotation pseudovector. 'As pointed out by Crawford, ${ }^{3}$ usirg classical Hamilton-Jacobi theory. Ehrenfest proved that $S$ is an adiabatic invariant, and postulated that $S$ is to be quantized as

$S=\left(n+n_{0}\right) 2 \pi n=\left(n+n_{0}\right) h$.

where $n=0,1,2, \ldots, \infty$, and $n_{0}$ is a constant adjusted to agree with experiment so as to give the correct ground state. Equation (8) with $n_{0}=1 / 2$ corresponds to the Bohr-Sommerfeld-wilson (aSW) quantization rule of the old quantum theory. In Eq.(7), the choice of $n_{0}=1 / 2$ by Huang is necessary and sufficient to ensure that the allowed perpendicular energy eigenvalues $E_{n}=(r$ - 1/2 His are of the harmonic oscillator type, and are in agreement with the earlier results of Landau. 13 Here, the cyclotron frequency $\omega_{D}=q B / \mu c$. If Huang would have chosen $n_{0}=0$ in agreement with Bloch, he wculd have cotained $E_{n}=n t \omega_{b}$, with no zero-point energy for the ground state, and this result will be in disagreement with that of Landau. The finite value of the grourd state zerc-point energy $\pi \omega_{b} / 2$ for a harmoric oscillator is characteristic of quantum mechanics and is related to the reiserberg's 
uncertainty principle. ${ }^{4,5}$ A somewhat similar view of assuming $n_{0}=1 / 2$ so as to give the correct Landau gyration ground state is also found in the paper by crawford. ${ }^{3}$ He also gives an excellent discussion of the adiabatic invariants associated with this cyclotron motion about the guiding center. Thus, the Landau gyration ground state has an energy $E_{0}=\pi \omega_{D} / 2$ and an action integral $S_{0}=\pi h=n / 2$, so that the ratio $\left(E_{n} / S_{n}\right)$ is the same for all Landau levels including the ground state. That 15, the Landau gyration ground state is the zero point gyration state with energy $\pi \omega_{D} / 2$ and an associated $z$-component of the angular momentum of $\pi / 2$.

Thus, according to Huang's "old" quantum theory, the orbital angular momentum of the Landau gyration states is $L=r \times p=r \times(\mu v+q A / c)$ and is not given by $L=r \times \mu v$, and the $z$-component of this orbital angular momentum takes half-odd-integral values, i.e., $L_{z}=(n+1 / 2)$ f. Also, according to this BSW quantization rule, the "allowed" orbits have radii satisfying the condition

$\left(r_{1}^{2}\right)_{n}=(2 \pi c / q B)(n+1 / 2)$

That is, the magnetic flux $\Phi_{n}$ enclosed by the $n$-th Landau orbit is

$\Phi_{n}=\pi\left(r_{1}^{2}\right)_{n} B=(2 \pi \pi c / q)(n+1 / 2)=(n c / q)(n+1 / 2)$,

and the associated $z$-component $M_{z}$ of the magnetic moment $M$ is

$\left(M_{z}\right)_{n}=q \omega_{D} \pi\left(r_{L}^{2}\right)_{n} / c=(q / \mu c)(n+1 / 2) \pi$.

Thus, from Eqs.(7) and (11), one finds that the g-value of these gyration states 
$g=M /(q / 2 \mu c) L=M_{z} /(q / 2 \mu c) L_{z}=2 \quad$ (for all $n$ ).

Thus we see that the Bohr-Sommerfeld-wilson quantization rule of the old quantum theory as used by Huang, yield a somewhat peculiar but nevertheless correct results for the Landau energy levels, the eigenvalues of the $z$-component of the canonical orbital angular momentum, and the g-value of these Landau gyration states. The g-value of two is of course entirely consistent with the nalf-odd-integral values for the angular momentum. What is really surprising is the fact that the $z$-component of the canonical obital angular momentum $L$ $=r \times p=r \times(\mu v+q A / C)$ takes half-odd-integral values. This is really tied to the fact that the force on the particle is purely velocity dependent and the motion is a circular motion about the guiding center in the $x-y$ plane, but the energy eigenvalues are those of the projected motion on one of these (either $x$ or $y$ ) axis only. That is, according to Landau, as far as the energy eigenvalues are concerned, this cyclotron motion about its guiding center is identical to that of a linear harmonic oscillator, i.e., $E_{n}=(n+1 / 2) \pi \omega_{D}$. This linear oscillator must of course have the zero-point energy of $\pi \omega_{D} / 2$ in order tc satisfy the Heisenberg's uncertainty principle. Since the motion in the $x-y$ plane is circular with a frequency $\omega_{D}$, it must also have a $z$-component of the angular momentum $L_{z}$ such that the ratio $\left(E_{n} / L_{z n}\right)$ is equal to the frequency $\omega_{D}$ for all the Landau levels $n=0,1,2 \ldots, \infty$. Thus, on physical grounds, this half-odd-integral values for the z-component of the canonical croital argular momentum is consistent with the Landau energy eigenvalues for this circular narmonic oscillator.

In the Heisenberg's matrix representation using the generalized abstract 
Hilbert space of Dirac's bra and ket vectors, the occurence of this half-odd-integral orbital angular momentum poses no real problem, since here we only require that the components of $r$ and $p$ satisfy the fundamental commutation relations of Eq.(2) and we do not require that $P$ be expressible as differential operator -in $\nabla$. However, if we assume that all spinless problems of quantum mechanics, regardless of whether the generalized potential $U$ is velocity dependent or not, must have a schrodinger's spatial representation and the canonical momentum operator $p$ must be expressible as the differential operator $-i \hbar \nabla$ in this $r$-representation, then the occurence of half-odd-integral values for the components of the orbital angular momentum $L=$ rxp poses a serious threat to the continuity and single valuedness for a spatial rotation through $2 \pi$ of the eigenfunctions $\psi_{n}(r, t)$. In this Schrodinger's $r$-representation, the spherical harmonics play an important role in the study of motions whose orbital angular momentum takes integral values of h. Thus, it would appear that some linear combination of Hermite polynomials in the $x$ and $y$ coordinates with a phase difference of $\pi / 2$ may play an important role in the study of motions whose orbital angular momentum takes half-odd-integral values of $h$, since the wave functions of the stationary states of a Landau particle in the nonsymmetric gauge are essentially the Hermite polynomials. 13

Alternatively, it is quite possible that this problem does not have ar. exactly equivalent schrodinger's spatial representation in the cylindrically symmetric gauge. It is not at all clear that, when the generalized potential $U$ of the problem under study is purely velocity dependent, whether one can indeed find an exact schrodinger's spatial representation, for example, withcut destrcying the physically obvious cylindrical symmetry of the problem in this particular case. There are of course problems in quantum mechanics that do not have a schrodinger's spatial representation. These problems always involve half-odd-integral angular momentum which are admissible to the definitions of 
Eqs.(3) and (5), Dut are certainly not admissible to that of Eq.(1) with $p=$ $-i \hbar \nabla$. For example, the Pauli spin Hamiltonian $H=\sigma \cdot \nabla \times A$ do not have a spatial representation in which one can write down a schrodinger equation. such a problem can only be handled as an eigenvalue problem in the Heisenberg's matrix representation in terms of the bra and ket spin vectors.

In order to get some more physical insight into this problem, let us take a detour and examine the similarity 15 between the "Zitterbewegung" motion of the Dirar's free particle Hamiltonian theory and the nonrelativistic Landau cyclotron motion of a spinless particle in the uniform $B$-field with $A(r)=$ $B \times r / 2$. The commutation relation between the operators of the velocity components $v_{j}$ (for $j=1,2$, and 3 ) of the Landau particle may be written 5,13

$\left[v_{j}, v_{k}\right]=i\left(q \operatorname{ctc} \mu^{2}\right) B_{1}, \quad(j, k, 1$ in cyclic order $)$

The Dirac Hamiltonian for a relativistic free spin $1 / 2$ particle is 4,15

$H=E=-\alpha \cdot p-\beta \mu c^{2}$.

where $\alpha_{j}$ for $j=1,2$, and 3 , and $\beta$ denote four Hermitian operators which acts on the spin variables alone, are independent of $r_{j}, P_{j}, t$, and $E$, and hence, commute with all of them. Nevertheless, for this Dirac free particle

$v_{j}=d r_{j} / d t=(i \hbar)^{-1}\left[r_{j}, H\right]=-c \alpha_{j}$

and since $\left[\alpha_{j}, \alpha_{k}\right]=2 i \sigma_{j} \quad(j, k, 1$ in cyclic order $)$, we get

$\left[v_{j}, v_{k}\right]=12 c^{2} \sigma_{1}^{\prime}(j, k, 1$ in cyclic order $)$, 
where the $\alpha_{j}$, and $\sigma_{j}^{\prime}$ for $j=1,2$, and 3 are taken here in the standard representation. 4,9,15 Comparing Eqs(13) and(16), we see that the Dirac's free-particle "Zitterbewegung" motion may be thought as some sort of Landau cyclotron motion in a "magnetic-type spinor field" $B_{j} \rightarrow\left(2 \mu^{2} c^{3} / q n\right) \sigma_{j}$ ' for $j=$ 1. 2, and 3. Then the angular frequency of the oscillatory motion $\omega_{b j}=$ $\left(q B_{j} / \mu c\right) \rightarrow\left(? \mu c^{2} / \pi\right) \sigma_{j}$. That is, the eigenvalues of the oscillation frequency are $\pm\left(2 \mu c^{2} / \pi\right)$. Since, $v_{j}=-c \alpha_{j} \rightarrow r \omega_{b j}$, the eigenvalues of the amplitude of the oscillation are $\pm(h / 2 \mu \mathrm{C})$. We have shown earlier that the Landau's zero point gyration state carries an orbital angular momentum of $\pm \pi / 2$ with a g-value of 2 . Thus, we see a striking similarity between the "Zicterbewegung" motion of the Dirac spin $1 / 2$ particle and the Landau's zero point gyration state. This similarity has been cast into a rigorous mathematical form by Barut and Zanghi. 16 Further, this striking analogy between the real Zitterbewegung of the spin 1/2 Dirac free particle and the zero point gyration state (with the canonical orbital angular momentum $\mathrm{h} / 2$ ) of the Landau particle may also shed some light on the degree of freeness of the Dirac free particle. 15

111. GaUge inVARIANCE AND the cORRECT ORBITAL ANGULAR MOMENTUM

We have seen that when the generalized potential $U$ in the Lagrangian $\mathcal{L}$ of any system is velocity dependent, then the canonical momentum $p=\partial \mathcal{L} / \partial \mathrm{v}$ is not equal to the mechanical momentum $\mu v$. Then, what is the croital angular momentum $L$ of such a system?. Is it $r \times p$, or is it rxuv?. For cur case $U=Q \Phi-q A \cdot v / c$, and hence $P=\mu v+q A / C$. From $w$ hat has been said 
earlier, it is clear that any angular momentum operator $J$ must satisfy the requirements of Eq.(3) or Eq.(5). In particular, if we satisfy Eq.(3), we will automatically satisfy Eq.(5) and vice versa. These Eqs.(3) and (5) are true in the abstract generalized Hilbert space of Dirac's bra and ket vectors of the Heisenberg's matrix representation. However, the orbital angular momentum $L$ of any system must be definable in the schrodinger's spatial representation with the aid of Eq.(1). But, Eq.(1) will satisfy the requirements of Eq.(3) if and only if the components of $r$ and $p$ satisfy the fundamental commutation. relations of Eq.(2), even if $P$ is not expressible as the differential operator -iti $\nabla$. Or course, if $P=-i \hbar \nabla$, the components of $P$ will automatically satisfy the requirements of Eq.(2). It is the fundamental assumption in the Hamiltonian formalisms of both the classical and quantum mechanics, the coordinate operator $r$ and the corresponding canonical momentum operator $p$ always satisfy the requirements of Eq.(2). In classical mechanics of ccurse the Poisson bracket $\{\cdot, \cdot\}$ replaces the commutator bracket as $(i / i \hbar)[\cdot, \cdot]$ in Eq.(2). Thus, it is clear that the canonical orbital angular momentum $L$ defined as $L=$ rxp, where $P$ is the canonical momentum, wiil clearly satisfy the requirements of Eqs. (1) to (5). In the Schrodinger's spatial representation, this canonical momentum $P=-i \pi \nabla$, and consequently the rctations around the $z$-axis, i.e., the changes in the azimuthal angle $\varphi$, are generated by the $z$-component of this canonical arigular momentum $L_{z}=$ $-i \hbar \partial / \partial \varphi$. This view is in complete agreement with those of Bloch, 11 schiff, 4 ard Huang: 12 but is in complete disagreement with those of Feynman 9 and wilczek. 10 Indeed, according to wilczek: "Rotations around the z-axis, i.e., the charges in the azimuthal angle $\varphi$ are generated by the covariant angular momentum: $L_{z}=-i \partial_{\varphi}-q A_{\varphi} . "$

This covarlant angular momentum $L_{F}$ of Wilczek and alsc of Feyrmar is related to the magnetic moment $M$ by a g-factor of one and not two, and is 
given by

$L_{F}=r \times \mu v=r \times[p-q A / c]=(2 \mu c / Q) M$.

According to Eqs.(3) or (5), this Feynman's angular momentum $L_{F}$ is not ar. acceptable angular momentum operator, since neither $L_{F} \times L_{F}=$ ifL $L_{F}$, nor is it a rotation operator. First of all, according to Eq.(13), we see that in a magnetic field, the operators of the velocity components $v_{j}, j=1,2$, and 3 , of a charged particle do not commute. This means that the particle cannot simultaneously have definite values of the velocity components in all three directions. This violates the basic requirements of Eq.(2). Second, from Eqs.(2) and (13), one car show that the components of $L_{F}$ satisfy the following commutation relations: 5

$\left[L_{F j}, L_{F k}\right]=\operatorname{in}\left(L_{F l}+\left(q r_{1}\right)(r \cdot B)\right\} \quad(j, k$, I in cyclic order $)$. (18)

When the B-field is not zero, it is clear from Eqs.(3) and (18) that the commitation relations of $L_{F}$ are completely different from those of ar angular momentim operator $J$. The operator $L_{F}$ therefore does not, a piori, pcsses the properties of an angular momenta. Hence, we conclude that Bloch's definition of the orbital angular momentum is the correct one, and the Feynman's definition is totally incorrect.

Let us now briefly examine the gauge invariance of the dynamical variables of a system in classical mechanics, and the gauge invariance of the corresponding Hermitian operators in quantum mechanics. Ir the gauge transformation of the electromagnetic field, the scalar ard the vector potentials transform as 
$\Phi^{\prime}(r, t) \rightarrow \Phi(r, t)-(1 / c) \partial \chi(r, t) / \partial t$, and

$A^{\prime}(r, t) \rightarrow A(r, t)+\nabla X(r, t)$

In both the classical and quantum mechanics, all physically meaningful results must not be modified when such a gauge transformation is performed,i.e., classical and quantum mechanics posses the property of gauge invariance. Bcth in the Lagrangian and in tha Newton's equations of motion formalisms of classical mechanics, the fundamental dynamical variables are the position $r$ and the velocity $v$, and these variables take on, at every instant, values independent of the gauge. These are usually referred ${ }^{5}$ to as true physical quantities or true physical dynamical variables. in this case, the feynman and Wilczek pseudovector $L_{F}=r \times \mu v$, although not an angular momentum operator, is a true physical quantity independent of the gauge. Then, the canonical momentum $P=\mu v+Q A / C$ and the canonical angular momentum pseudovector $L$ = rxp, although a true angular momentum operator, are gauge dependent. These gauge dependent dynamical variables in classical mechanics are usually referred ${ }^{5}$ to as non-physical quantities or non-physical dynamical variables.

However, in quantum mechanics which is based on the Hamiltorian formalism, the fundamental Hermitian operators which replace the classical dyramical variables are the position and the canonical momentum operators $r$ and $p$, respectively. These operators and the associated commutation relations of Eq.(2) are the same in all the gauges. When the problem under study has a Schrodinger's spatial representation, $p=-i \pi \nabla$ in all gauges. Thus, in quarturr. mecharics, the canonical angular momentum operator $L=r \times p$ is the same ir all gauges. Thus, the relations of Eqs.(1) to (5) are gauge independert. out, the velocity cperator $V=\mu^{-1}[p-q A / C]$ and the reynman-wilczek cperatcr $L_{F}=$ 
$r \times \mu v$, on the other hand, depend on the gauge chosen. In general, the Hermitian operator of quantum mechanics, corresponding to a true physical quantity (or a true physical dynamical variable) of classical mechanics, depends on the gauge chosen.

In quantum mechanics, under the gauge transformation of Eq.(3), the Dirac's ket vector transforms as

$\mid n>^{\prime} \rightarrow[\exp \{i(q / f c) \times(r, t)] \mid n>$.

That is, for the wave function, the gauge transformation corresponds to a phase change which varies from one point to another, and is not therefore a global phase factor [as in Eqs.(45), (47), and (48), for example]. Infact, one can show that $X$ is not single valued but changes by

$\Delta X=\oint A \cdot d l=\oint \nabla X \cdot d l=\iint B \cdot d s=\Phi$

whenever one goes around a closed loop. This phase change $\Delta X$ around a closed ICOP is proportional to the Aharonov-Bonm flux enclosed by the closed path.2.21 Thus, in quantum mechanics, the vector potential is a measurable quanilty via the Aharonov-Bohm phase change in the wave functions; and as Feynman ${ }^{2}$ says it is a real field. Feynman defines the "real field" as a set of numbers we specify in such a way that what happens at a point depends only on the rumbers at that point.

IV. CLASSICAL CYCLOTRON MOTION ABOUT THE GUIDING CENTER

Let us now examine briefly the classical cyclotron moticn abcut the 
guiding center of a charged particle of charge $q$ and mass $\mu$ in a uniform magnetic field $\mathrm{B}=\mathrm{Bi}_{2}$ whose vector potential in the cylindrically symmetric gauge is $A(r)=A_{\perp}\left(r_{\perp}\right)=B \times r / 2=(B / 2)\left(i_{2} \times r_{\perp}\right)$, where $\perp$ refers to directions perpendicular to the liniform B-field. The equation of motion of such a particie is 7

$d v_{\perp} / d t=\omega_{D}\left(v \times i_{Z}\right)$

where the cyclotron frequency $\omega_{D}=q B / \mu c$. The motion described by Eq.(22) is a circular motion in the plane perpendicular to $B$. The solution for $v_{\perp}$ is easily shown to be

$v_{\perp}(t)=\omega_{D} r_{\perp D}\left(i_{x}-i i_{y}\right) e^{-i \omega_{D} t}$

where $r_{\perp D}$ is the gyration radius. The convention is that the real part of the equation is to be taken. Then one can see that Eq.(23) represents a counterclockwise rotation for a positive charge $q$ when viewed in the direction of $B$. Another integration yields the radius vector of the particle about its guiding center as

$r_{-}(t)=i r_{\perp D}\left(i_{x}-i i_{y}\right) e^{-i \omega_{D} t}$

Thus, from Eqs.(23) and (24), we get

$v_{-}=-\omega_{D}\left(i_{Z} \times r_{1}\right)$

Herce, using Eq.(25), we get 
$P_{\perp}=\mu v_{\perp}+q A_{\perp} / c=\mu v_{\perp}+(\mu / 2)\left(\omega_{D} i_{2} \times r_{\perp}\right)=\mu v_{\perp}-\mu v_{\perp} / 2=\mu v_{\perp} / 2$

Thus, in this cylindrically symmetric gauge, the $z$-component of the canonical orbital angular momentum is

$L_{z} i_{Z}=r_{\perp} \times P_{\perp}=(\mu / 2) r_{\perp} \times v_{\perp}$.

But the $z$-component of the magnetic moment $M$ may be written

$M_{z} i_{z}=(q / 2 \mu c) r_{\perp} \times \mu v_{\perp}$.

Thus, from Eqs.(27) and (28), we get the classical canonical g-value as

$g=M_{z}^{\prime}(q / 2 \mu c) L_{z}=2$.

We see then, even classically, the canonical g-value of the cyclotron motion abcut the guiding center is two and not one. This of course is due to the peculiar relation of Eq.(26) between the canunical momentum $p$ and the mechanical momentum $\mu v$, which in turn is due to the peculiar velocity dependent force in the equation of motion of Eq.(22). That is, as pointed out by crawford ${ }^{3}$ the contribution to the action integral $s$ from the potential momentum $Q A / C$ has half the magnitude and opposite in sign of the contribution of the $\mu v$-momentum. Stated differently, the diamagnetic coritribution to the magnetic moment which is due to the Aharonov-Bchm phase integral around the orbit is exactly equal to the paramagnetic coritributicr tc the magnetic momert which is due to the canonical angular momentum. 28 
V. LANDAU GYRATION STATES IN THE HEISENBERG'S MATRIX REPRESENTATION

We again consider the motion of a spinless particle of charge $q$ and mass $\mu$ in a uniform external magnetic field $B=B i_{z}$. Let $r, v$, and $p$ be, respectively, the position, velocity, and canonical momentum vectors of the particle. The vector potential $A(r)$ for such a uniform $B$-field may be written

$A(r)=A_{\perp}\left(r_{L}\right)=(1 / 2) B \times r=(B / 2) i_{Z} \times r_{\perp}$.

The Hamiltonian describing the motion of the particle is

$H=(p-q A / C)^{2} / 2 \mu=H_{\perp}+H_{\|}$.

where

$H_{-}=\left(p_{\perp}-q A / C\right)^{2} / 2 \mu=\left(p_{x} i_{x}+p_{y} i_{y}-q A / C\right)^{2} / 2 \mu$

and

$H_{\|}=P_{\|}^{2} / 2 \mu=P_{z}^{2} / 2 \mu$.

Here, - and II refer to directions perpendicular and parallel, respectively, to the uniform magnetic field $B=B i_{z}$. Since $A \cdot i_{z}=c$ for the uriform magnetic field $B=B i_{Z}$, we see that the total Hamiltoniar ( $H$ ) separates irto two parts, one describing the cyclotron motion of the charged spirless particle in a plane perpendicular to the $\mathrm{B}$-field $\left(\mathrm{H}_{\perp}\right)$ and the cther cescrioing its free 
motion parallel to the $B$-field. It is shown eisewhere 5.13 that this $H_{\perp}$ can be written in a form which is analogous to that of a Hamiltonian of a one-dimensional harmonic oscillator. This equivalence to a one-dimensional harmonic oscillator was first shown by Landaul3 in the Schrodinger's $r$-representation using the rion-symmetric gauge where $A_{x}=-B y, A_{y}=A_{z}=0$. In this Landau's Schrodinger equation formalism, the problem under study does not exhibit the cylindrical symmetry it actually possess. Also one can show 5 that, in this particular cylindrically symmetric gauge of Eq.(30), this same operator $H_{\perp}$ is simply related to the Hamiltonian of a two-dimensional harmonic oscillator. These two results (i.e., the simultaneous similarity to both the one-dimensional and the two-dimensional harmonic oscillators) are not contradictory; they simply correspond to two different decomposition of the same Hamiltonian, which must obviously lead to the same physical conclusions. However, one must not lose sight of the ract that the Hamiltonian $H_{\perp}$ involves a physical problem which is completely different from that of a two-dimensional harmonic oscillator: the charged particle is subjected to a vector potential (describing a uniform magnetic field) and not a harmonic scalar potential (which would describe, for example, a non-uniform electric field). It so happens that, in the gauge chosen, the effects of the magnetic field can be likenes to those of a fictitious harmonic scalar potertial. classically we know that the particle motion is a circle in a plane perpendicular to the $B-f i e l d$, and the $x$ and $y$ coordinates of the particle describe linear harmonic motion with a $90^{\circ}$ phase difference between them. Although the projected motions on the $x$ and $y$ axes are formally identical tc that of a linear harmonic oscillator, it should be emphasized that the moticn of the particle under study is determined by a velocity-dependent potential $(q A \cdot v / c)$ in its Lagrangian [or its velocity-dependent force ( $q v \times B / c)$ ]. For these reasons one can show 3 that the contribution to the "action integral" $s$ of the "pctential momentum" $Q A / C$ has half the magnitude and opposite in sign to the 
contribution of the " $\mu v$ momentum". This in turn is responsible for the g-value of two and not one for the cyclotron motion atout the guiding center under study, and this behavior is quite different from that of a two-dimensional harmonic oscillator. 5

In the spirit of $D$ : ac's suggestion, 17 we will now take a detour and examine this problem (or the cyclotron motion about its guiding center) in the Heisenterg's matrix representation via its equations of motion. This method of analysis will explicitly exhibit the cylindrical symmetry about the uniform magnetic field $B=B i_{Z}$. The Heisenberg's operator equation of motion for the perpendicular velocity operator $v_{\perp}=v_{x} i_{x}+v_{y} i_{y}$ is given by

$\dot{v}_{\perp}=(! / \Re)\left[H_{\perp}, v_{\perp}\right]=(i / \pi)\left(H_{\perp} v_{\perp}-v_{\perp} H_{\perp}\right)$.

Since the Lniform magnetic field $B=B i_{Z}$ commutes with $v_{\perp}$, the quantum mechanical symmetric average ${ }^{4}\left(v_{\perp} \times B-B \times v_{\perp}\right) / 2=v_{\perp} \times B$, and hence the vector equation of the perpendicular motion is 7

$\dot{v}_{\perp}=\left(q / \mu c ; v_{\perp} \times B\right)=\omega_{D}\left(v_{\perp} \times i_{Z}\right)$,

where $\omega_{D}=(q B / \mu C)$ is the cyclotron frequency. We now chocse the perpendicular energy representation in which $H_{\perp}$ is diagonal. That is, $H_{\perp m n}=$ $E_{n} \delta_{m n}$ [or $H_{-}\left|n>=\sigma_{n}\right| n>$ and $\langle m| n>=\delta_{m n}$ ]. Then in terms of the matrix elements, Eqs.(34) and (35) become

$\dot{v}_{-m n}=(1 / A)\left(E_{m}-E_{n}\right) v_{I m n}$ 
and

$\dot{v}_{1 m n}=\omega_{D}\left(v_{\perp} \times i_{Z}\right)_{m n} \quad$

respectively. From Eqs.(36) and (37) we get

$\left(E_{m}-E_{n}+\hbar \omega_{D}\right) v_{+m n}=0$

$\left(E_{m}-E_{n}-\gamma \omega_{D}\right) v_{-m n}=0 \quad$.

where

$v_{ \pm}=2^{-1 / 2}\left(v_{x} \pm i v_{y}\right)$

It can be shown that the following commutation relations are true: 5,13

$\left[v_{-}, v_{-}\right]_{m n}=-i\left[v_{x}, v_{y}\right]_{m n}=\left(\hbar \omega_{D} / \mu\right) \delta_{m n}$

$\left[v_{x}, v_{z}\right]_{m n}=\left[v_{y}, v_{z}\right]_{m n}=0$

The perpendicular velocity operator $v_{\perp}$ is given by

$v_{\perp}=d r_{\perp} / d t=(i / A)\left[H_{\perp}, r_{\perp}\right]=\left(p_{\perp}-q A_{\perp} / c\right) / \mu$

Thus on making use of Eqs.(41) and (42) in Eq.(32) we get

$r_{-m n}=\mu\left(v_{+} v_{-}\right) m n-\left(\hbar \omega_{D} / 2\right) \delta_{m n}=\mu\left(v_{-} v_{+}\right)_{m n}+\left(\hbar \omega_{D} / 2\right) \delta_{m r}$ 
From Eqs.(38) to (43) we get, by exact analogy to the customary theory of a linear harmonic oscillator, $4,5,13$ the energy level spectrum and the nonzero matrix elements of $v_{+}$and $v_{-}$as

$E_{n}=H_{\perp n n}=(n+1 / 2) \pi \omega_{D}$

where $n=0,1,2, \ldots, \infty$,

$v_{+} n-1, n=\left(v_{-} n, n-1\right)^{*}=\left(\pi \omega_{b} / \mu\right)^{1 / 2} n^{1 / 2} e^{i \varphi_{n}}$

where $\varphi_{n}$ is an arbitrary phase factor and the asterisk means the complex conjugate. Since

$v_{\perp m n}=\dot{r}_{\perp m n}=(i / \pi)\left[H_{\perp}, r_{\perp}\right]_{m n}=1 \omega_{D}(m-n) r_{\perp m n}$.

from Eq.(45) we get the nonzero matrix elements of the operators $r_{+}$and $r_{\text {_ }}$ as

$r \cdot n-1, n=(r-n, n-1)^{*}=i\left[\left(\hbar / \mu \omega_{D}\right)=(h c / q B)\right]^{1 / 2} n^{1 / 2} e^{i \varphi} n$,

where $r_{ \pm}=2^{-1 / 2}(x \pm 1 y)$. From Eqs.(45) and (47), the nonzero matrix elements of the perpendicular velocity and position operators are

$$
\begin{aligned}
& v_{x, n, r-1}=\left(v_{x} n-1, n\right)^{*}=-i v_{y}, n-1=\left(i v_{y} n-1, n\right)^{*}=\left(\hbar \omega_{D} / 2 \mu\right)^{1 / 2} n^{1 / 2} e^{-1 \varphi_{r}}, \\
& x_{n, n-1}=\left(x_{n-1, n}\right)^{*}=-i y_{n, n-1}=\left(i y_{n-1, n}\right)^{*}=-i\left(\pi / 2 \mu \omega_{D}\right)^{1 / 2} n^{1 / 2} e^{-i \varphi_{n} .(48)}
\end{aligned}
$$


From Eqs.(30) and (42), it is relatively easy to see that

$P_{\perp}=P_{x} i_{x}+P_{y} i_{y}=\mu\left(v_{x}-y \omega_{D} / 2\right) i_{x}+\mu\left(v_{y}+x \omega_{D} / 2\right) i_{y}$.

and

$p_{ \pm}=2^{-1 / 2}\left(p_{x} \pm i p_{y}\right)=\mu v_{ \pm} \pm i\left(\mu \omega_{D} / 2\right) r_{ \pm}$.

Thus, on making use of Eqs.(45), (47), and (48) in Eqs.(49) and (50), one can easily obtain the nonzero matrix elements of the perpendicular canonical momentum operator $P_{\perp}$. Since, $\pm i=\exp ( \pm i \pi / 2)$, it is seen from Eq.(48) that the particle motion about the guiding center is a circle in a plane perpendicular to the $B-f i e l d$ and the $x$ and $y$ coordinates of the particle describe linear harmonic motions with a phase difference of $\pi / 2$. Thus, in sharp contrast to the Landau's Schrodinger equation analysis in the $r$-representation, 13 our analysis in terms of the equations of motion in the Heisenberg matrix representation yields the expected classical picture of the particle motion about its guiding center.

For any volume distribution of current the magnetic moment $M$ is defined in Gaussian units as

$M=(1 / 2 c) \int(r \times j) d V$,

where $d V$ is the volume element, and $j$ is the current density. For a plane lcop of thin wire of cross-sectional area dS in which a current 1 is flowing. $j d V=j d S d l=1 d l$, where $d l$ is an element of length along the directior of the current flow. Then M of Eq.(51) becomes 
$M=(1 / 2 c) \oint(r \times d I)=(A \mid / c) n$.

where the area of the loop $A=\oint(r \times d) / 2$, and $n$ denotes a unit vector normal to the plane of the loop. For our problem, $n=-i_{Z}, 1=q \omega_{D}$, and $A=$ $\left(\pi r_{\perp}^{2}\right)=\pi\left[\left(r_{+} r_{-}\right)+\left(r_{-} r_{+}\right)\right]$. Thus, on making use of Eq.(47), we get

$\left(M_{z}\right)_{n n} i_{z}=\left\langle n\left|M \cdot i_{z}\right| n>i_{z}=\left(-i_{z}\right)(q n / \mu c)(n+1 / 2)\right.$.

We note that this result of Eq.(53) can also be obtained trivially by making use of the Feynman's theorem, according to which 28

$\left(M_{Z}\right)_{n n}=\left\langle n\left|M_{Z}\right| n\right\rangle=\left\langle n\left|-\partial H_{\perp} / \partial B\right| n\right\rangle=-\partial E_{n} / \partial B=-(q A / \mu C)(n+1 / 2),(54)$

where we have made use of Eq.(44). Since,

$M_{Z} i_{Z}=(q / 2 \mu c)\left(r_{\perp} \times \mu v_{\perp}\right)=(q / 2 \mu c)\left[r_{\perp} \times\left(p_{\perp}-q A_{\perp} / c\right)\right]=(q / 2 \mu c)\left[r_{\perp} \times p_{\perp}-\right.$ $\left.(q B / 2 c)\left(r_{\perp} \times i_{Z} \times r_{\perp}\right)\right]=(q / 2 \mu c) L_{Z} i_{Z}-(q / 2 \mu c)(q B / 2 c)\left(r_{\perp} \cdot r_{\perp}\right) i_{Z} \cdot(55)$

where we have made use of Eq.(30), the relation $\mu v=p-q A / C$, Eq.(28), and the fact that $\left(i_{z} \cdot r_{\perp}\right)=0$. The last equality of Eq.(55) exhibits the paramagnetic and the diamagnetic contributions explicitely. Thus, from Eq.(55) we get

$\left(L_{z}\right)_{n \Gamma}=(2 \mu c / q)\left(M_{z}\right)_{n} \cdot(q B / 2 c)\left[\left(r_{-} r_{-}\right)+\left(r_{-} r_{+}\right)\right]_{n n}$

$$
=-2(n+1 / 2) \pi+(n+1 / 2) \pi=-(n-1 / 2) \pi .
$$


where we have made use of Eqs.(47) and (54). From Eq.(47), the eigenvalues of the magnetic flux of these gyration states are

$\Phi_{n n}=\pi\left(r_{\perp} \cdot r_{\perp}\right)_{n n} B=\pi B\left[\left(r_{+} r_{-}\right)+\left(r-r_{+}\right)\right]_{n n}=(n c / q)(n+1 / 2) .(57)$

From Eqs.(54) and (56), we see that the g-value or these Landau gyration states is $g=\left[M_{z} /(q / 2 \mu c) L_{z}\right]=2$ for all $n$. As seen from Eqs.(53) to (56), this $g=$ 2 is a consequence of the fact that the diamagnetic and the paramagnetic contributions to the magnetic moment are interdependent. Indeed, the diamagnetic contribution is exactly equal to the paramagnetic one, and is related to the Aharonov-Bohm magnetic flux. Thus we see that the rigorous analysis of the cyclotron motion about its guiding center using the cylindrically symmetric gauge in the Heisenberg matrix representation via Dirac's suggestion of using its equation of motion, yield the same resuls as those previously obtained by Huangl2 using the "old" quantum theory with the Bohr-Sommerfeld-Wilson quantization condition for the action integral $S$ with the choice of $n_{0}=1 / 2$ so as to give the correct Landau ground state. It is interesting to note that in the old quantum theory the quantization of the action integral $S$, or equivalently, the components of the orbital angular momentum $L$, is the most fundamental entity. Then the quantization of energy, magnetic moment, and the magnetic flux follow as a consequence of the s-quantization. While in the modern quantum theory the system Hamiltonian is the most fundamental. In both the nonrelativistic and in the manifestly Lcrentz noncovariant but Lorentz invariant 15,26 Dirac's relativistic theories, the Hamiltonian of the system is the total energy $E$ of this system. Hence, in the modern quantum theory, the quantization of the energy $E$ is the furdamental entity. Then the quantization of the angular momentum, magnetic moment and the magnetic flux follow as a consequence of the E-quartizaticr. It is also interesting to note from Eqs.(10) and (57) that the magnetic flux 
associated with these Landau gyration states is quantized in steps of (hc/q), but with a zero-point flux of $(\mathrm{hc} / 2 \mathrm{q})$. We also pointed out the striking similarity Detween the Dirac free particle zitterbewegung motion and the ground state of the Landau cyclotron motion, so that spin is the orbital angular momentum of this Landau zero-point gyration state or the zitterbewegung.16,15 This is suggestive of the Onsager's idea that the flux quantization in units of this zero-point gyration value of (hc/2q) might be an intrinsic property of the electromagnetic field-charged particle interaction. 20 We know that the zero-point vibrations of the quantized radiation field determines the change in the radiative self-energy of quantum states which are responsible to the Lamb shift and the schwinger $(g-2)$ value. 29,30 it is therefore not unreasonable to speculate on the link between the intrinsic flux quantization unit and the flux associated with the Landau zero-point gyration state. It is not clear whether this has any implications to the concept of "electron pairing" in the Bardeen-Cooper-schrieffer (BCS) theory of low temperature superconduc ivity. 19 we will come back to examine this link in some detail later.

VI. FLUX QUANTIZATION AND THE UNIT OF MAGNETIC FLUX

It has been shown by London, 31 onsager, 20 and Byers and Yang 21 that the magnetic flux embraced by a superconducting ring (i.e., a ring of noninteracting free electron gas) ought to be quantized in units of $h \mathrm{c} / \mathrm{q}$, where $\mathrm{q}$ is the magritude of the electronic charge. However, the magnetic flux through a superconducting ring has been found experimentally 18 to be quantized in units of hc/2q. The experimental discovery of steps just this size has been claimed to provide a convincing direct proof for the pairing of electrons as predicted by the theory of Bardeen, Cooper and schrieffer (BCS). 19 This pairing is due to 
the interaction of the conduction electrons in the superconductive state, and consequently the carriers of electricity in the superconductor has twice the charge of the electron.2.19 it is our aim here to present a somewhat controversial and speculative counter arguement to show that the discovery of the rundamental unit of flux quantum as $\mathrm{hc} / 2 \mathrm{q}$ instead of $\mathrm{hc} / \mathrm{q}$ need not necessarily imply the existence of "electron pairing" in the superconductive state.

For this purpose, let us consider a solid cylinder of finite radius $R$ placed in a uniform magnetic field $B=B i_{z}$ with its axis parallel to the B-field. Let this cylinder contain $N$ electrons and we will assume that these $N$ electrons form a noninteracting free electron gas, similar to that in a superconductor. We will further assume that the temperature $T$ of this cylinder is low enough that we are not in the Landau diamagnetic regime, but are in the De Hass-Van Alphen regime. It is clear from Eqs.(10) and (57) that the magnetic flux associated with the $n$-th Landau gyration state is $\Phi_{n}=($ hc/q) $(n+1 / 2)$. That is, the flux enclosed by the successive Landau orbits increases in steps of $\mathrm{hc} / \mathrm{q}$ starting from the ground state value of hc/2q. Following Huang. 12 it is relatively easy to show that each of these Landau gyration states are continously degenerate, and the degeneracy of the energy level $E_{n}$ is the same for all $n$ and is given by

$G=\left(R^{2} q B / 2 \pi c\right)$,

since the cross-sectional area of the cylinder is $\pi R^{2}$. Physically this degeneracy arises from the fact that the Landau orbit in the magnetic fiels has the same energy, wherever its guiding center. Since we have $N$ free electrons Ir a cylinder of radius $R$, let $j$ be the largest integer for which $\left(r_{-}^{2}\right)$, of Eq.(9) is less than or equal to $R^{2}, 1$. e., 
$\left(r_{\perp}^{2}\right)_{j}=(2 \pi c / Q B)(j+1 / 2) \leq R^{2}$.

That 15, only the levels $0,1,2, \ldots . j$ are to be occupied by the available $N$ free electrons. It is clear from Eq.(59) that the value of the magnetic field $B_{j}$ at which the j-th Landau orbit will become available for occupation by the $N$ free electrons under study (i.e., the value or the $B$ field at which the radius of the $j$-th Landau orbit will equal $R$ ) is

$B_{j}=\left(\pi c / Q R^{2}\right)(2 j+1)$.

rerce for $1=0,1,2$, and 3, for example, the values of $B$ field at which the magnetic flux $\Phi$; will make a quantum jump are: $B_{C}=\left(A c / a R^{2}\right), B_{1}=$ $3\left(\pi c / Q R^{2}\right), B_{2}=5\left(\pi c / Q R^{2}\right)$, and $B_{3}=7\left(\pi c / Q R^{2}\right)$, respectively. That is, the ratic cf the fields at which the magnetic flux jumps will occur are approximately 1, 3, 5, and 7. This ratio of the critical fields has a striking similarity tc those observed experimentally for a somewhat different problem. 18 These experiments on quantized flux were done with hollow superconducting cylinders. while the result of EQ.(60) is derived only for a solid cylinder of a free electron gas. Nevertheless, this accidental agreement in the ratic of critical fields is somewhat remarkable.

We have $N$ electrons to be distributed among $(j+1)$ Lardau levels each cf degeneracy $G$. Hence the number of electrons $N_{r}$ in the $n$-th level is

$N_{r} \propto \begin{cases}N G e^{-E_{r} / K T} & \text { for } r \leq 1 \\ 0 & \text { for } n \geq 1\end{cases}$ 
where $T$ is the temperature and $k$ is the Boltzmann constant. We will assume that this temperature $T$ is low enough so that we are in the De Hass-Van Alphen regime and not in the Landau diamagnetic regime, but the highest occupied level $j$ is such that $E_{j}=(j+1 / 2) \pi \omega_{D}=(\operatorname{are} / \mu \mathrm{C})(j+1 / 2)$ $\ll k T$, so that $\exp \left(-E_{n} / K T\right) \approx 1$ for all $n \leq j$ in Eq.(61). Since, $\Sigma_{n=0}{ }^{j} N_{n}=$ N. Eq.(61) Decomes

$N_{\Gamma} \approx \begin{cases}N /(j+1) & \text { for } n \leq 1 \\ 0 & \text { for } n \geq j\end{cases}$

Wher the j-th Landau orbit is the largest orbit inside the cylinder of radius R, from Eqs.(10) or (57) and Eq.(62), we get the average magnetic flux $\langle\Phi,>$ nside this cylinder of radius $R$ as

$<\Phi_{j}>(1 / N) \Sigma_{n=0}\left(N_{n} \Phi_{n}\right)=(h c / q)(j+1)^{-1} \sum_{n=0} j(n+1 / 2)$

$=(h c / q)(j+1)^{-1}[(j+1)(j+1 / 2+1 / 2) / 2]=(h c / 2 q)(j+1) .(63)$

Thus, for $1=0,1,2$, and 3 for example, the average magnetic flux $\left\langle\Phi_{j}\right\rangle$ of Eq.(63) take the values $\left\langle\Phi_{0}\right\rangle \approx(h c / 2 q),\left\langle\Phi_{1}\right\rangle \approx 2(h c / 2 q),\left\langle\Phi_{2}\right\rangle \approx 3(h c / 2 q)$, and $\left\langle\Phi_{z}\right\rangle \approx 4(h c / 2 q)$, respectively. Since, for $B<B_{C}$ there is no Landau level irside the cylinder of radius $R$, the average magnetic flux $\left\langle\Phi_{1}\right\rangle$ will take the values 
$<\Phi_{j}>\approx \begin{array}{ccl}0 & \text { for } & 0<B<B_{0} \approx\left(\pi c / q R^{2}\right) \\ (h c / 2 q) & \text { for } & B_{0}<B<B_{1} \approx 3\left(\pi c / q R^{2}\right) \\ 2(h c / 2 q) & \text { for } & B_{1}<B<B_{2} \approx 5\left(\pi c / q R^{2}\right) \\ 3(h c / 2 q) & \text { for } & B_{2}<B<B_{3} \approx 7\left(\pi c / q R^{2}\right) \\ \cdot & & \\ \cdot & & \\ (j+1)(h c / 2 q) & \text { for } & B_{j-1}<B<B_{j} \approx(2 j+1)\left(\pi c / q R^{2}\right)\end{array}$

Here again, the result of Eq.(64) has a striking similarity to those observed experimentally for a somewhat different problem. That is, this result of Eq.(64) for a solid cylinder has a striking similarity to the experimental results obtained with hollow superconducting cylinders. Further, what is measured in the experiments is the trapped flux, while these calculations refer to the average flux enclosed by the complete Landau gyration orbits which can be accommodated inside this solid cylinder. It is not unreasonable to assume that only these complete Landau gyration orbit fluxes enclosed inside this solid cylinder can be frozen in or trapped when the metal cylinder makes the transition to the superconductive state as its temperature is lowered. In any case, we find that the average magnetic flux $\left\langle\Phi_{j}\right\rangle$ within a finite radius cylinder of a noninteracting free electron gas in an axially symmetric uniform

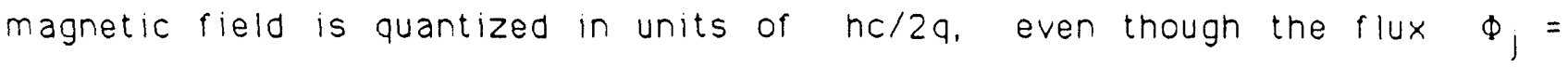
$(h \mathrm{~h} / \mathrm{q})(j-1 / 2)$ enclosed by the Landau orbits steps by hc/a. At low temperatures, when the energy of the system has been reduced very,very low, instead of a large number of states being involved, only a very, very small rumber near the ground state are involved. Under these circumstances the quantum mechanical character of that ground state can appear on a macroscopic scale. This is the reason that the average flux $\left\langle\Phi_{j}\right\rangle=($ hc/2q) $(j+1)$ is 


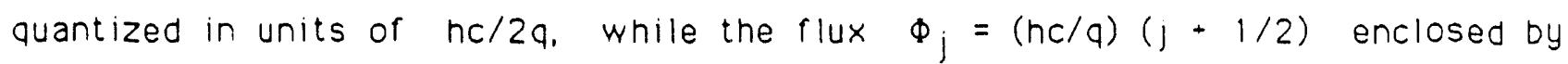
the successive Landau orbits steps in units of hc/q. Thus the experimental discovery of the fundamental unit of flux quantum as hc/2q instead of as hc/a need not necessarily imply the existence of "electron pairing" in the superconductive state, as predicted by the BCS theory. It could very well be that this unit of flux is tied to the fact that the system is not in the Landau diamagnetic regime Dut is in the De Hass-Van Alphen regime of temperatures, as in the case studied here. However, what is discussed here is not a theory of superconductivity, and there is nothing in our formalism that will predict the field expulsion and the start up of the supercurrent, i.e., the Meissner effect, for example.

VII. CHARGE-MONOPOLE COMPOSITES OR DYONS, SPIN AND STATISTICS

We have seen that according to wilczek, rotations around the z-axis, i.e., chanyes in the azimuthal angle $\varphi$ are generated by the $z$-component of the Feynman's "angular momentum operator" $L_{F}=r \times \mu v=r \times p-(q / c) r \times A$. This is really a magnetic moment operator, and is in no sense an angular momentum operator. In classical mechanics this pseudovector $L_{F}$ is a true physical dynamical variable, independent of the gauge. But in quantum mechanics, all Hermitian operators corresponding to these true physical dynamical variables depend on the gauge chosen. Thus, in the Hamiltonian quantum mechanics, the canonical momentum $\quad D=\mu v+Q A / C$ and the canonical crbital argular momentum cperator $L=$ rxp are the same in all the gauges, and hence are gauge covariant operators. This is due to the fact that the commutaticr relations of Eq.(2) and (3) are the same in all gauges. Using this Feynman's "argular momentum operator" in the schrodinger's spatial representation where 
$P=-i n \nabla$, one obtains the relation of Eq.(6). Here, of course, we have assumed a priori that the problem under study, regardless of the fact that the generalized potential $U=-Q A \cdot V / C$ of this problem is entirely velocity dependent, has indeed an exact Schrodinger's spatial representation. If we further assume that the azimuthal dependence of the particle wave function is $\Psi_{m} \propto \exp (\operatorname{im} \varphi), m$ integer for continuity and single valuedness for rotations through $2 \pi$, then Eq.(6) yield the wilczek 10 result

$L_{F z}=$ (integer) $h-q \Phi / 2 \pi c$.

If now we assume that a single magnetic monopole of magnetic charge $a_{\mathrm{m}}$ existed, then $\nabla \cdot B=4 \pi a_{m}$. Then by Gauss's divergence theorem $\int \nabla \cdot B$ OV $=$ $\int B \cdot d S$. Thus, the total flux $\Phi=4 \pi a_{m}$. Using these, wilczek argues that for a charged particle orbiting infinitesimally above this pole the flux is $2 \pi a_{m}$ while for a particle infinitesimally below it is $-2 \pi q_{m}$. The corresponding shifts in Eq.(65) are $L_{F z}=$ (integer) $\pi-\left( \pm q q_{m} / c\right)$. For these two spectra of ' $L F z$ values to agree one must have $\left(q a_{m} / c\right)=[(1 / 2)$ - integer $]$. This is the Dirac quantization condition. 24

In a profound paper on a magnetic model of matter schwinger 22 presents a conceivable dynamical interpretation of the subnuclear world. This he erects on the basis of the speculative but theoretically well-founded hypothesis that electric and magnetic charge car reside on a single particle. He names these dual-charged particles as dyons. Here, he first shows that the generalized Maxwell's equations are invariant for rotation through an arbitrary argle $\theta$, ard the cbserved absence of magnetic charge is described as the ccexistence of electric and magnetic charge in the universal ratio $a_{m} / q=\tan \theta$. Then he 
considers the nonrelativistic behavior of a particle with mass $\mu$, carrying an electric charge $q_{1}$ and magnetic charge $q_{m}$ l. which moves with velocity $v$ in the field of a stationary body that possesses charges $q_{2}$ and $a_{m}$. From Newton's equation of motion, he recognizes the conserved angular momentum vector as

$J=r \times \mu v-\left(a_{1} a_{m 2}-a_{2} a_{m 1}\right) r / c r$

The quantization of the component of this angular momentum along the connecting line of the particles then gives the charge quantization condition

$\left[\left(a_{1} a_{m 2}-a_{2} a_{m} !\right) / c\right]=2 n n$.

where $n$ is an integer. According to schwinger, if there are dual-charged particles, rather than electrically charged particles and magnetically charged particles, the quantization of $99_{\mathrm{m}} / \mathrm{hc}$ must be even integers. But according to Dirac and Wilczek $99 \mathrm{~m} / \mathrm{hc}=1 / 2$ + integer. It appears that witten 32 is comfortable with both these points of view, i.e., either integer or half-integer value for zero vacuum angle $\theta$. Dirac ${ }^{4}$ arrives at half-integral values simply by quantizing the uncertainty in the electrodynamic action integral according to Bohr's quantization condition of Eqs.(7) and (8) with $n_{0}=0$, or equ valently, by using the condition that the wavefunction must be single valued. Wilczek's explanation 10 of the relationship between the Dirac condition and the spin shift of $1 / 2$ unit, in terins of the continuity in the $L_{F Z}$ spectrum is also very appealing. However, schwinger 22 also gives a simple argument in behalf of integer quantization. This argument is based on the fact that matter is normally magnetically neutral, and when the electromagnetic angular momentum 
of Eq.i66) with $\mathrm{a}_{2}=0$ is made to vanish by a compensating magnetic charge $-q_{m 2}$ brought in from infinity to the origin so as to neutralize the magnetic charge $a_{m 2}$ at the origin, the net mechanical angular momentum $r \times \mu v$ of Eq.(66) must take only integral values. On shiftirg the viewpoint between the physically equivalent two-particle system and the three-particle system with an infinitely remote compensating charge, paradoxical transitions between (integer + 1/2) and integer values of the angular momentum will be avoided if $9 a_{\mathrm{m}} / \mathrm{hc}$ is restricted to iniegral values. These schwinger's arguments rely on quantizing the action integral based on classical Newton's equations of motion. When one has a non-zero vector potential $A$ with a vanishing $B$, then these arguments for rxuv may be incorrect. We wish to caution the readers about such situations. Furthermore, schwinger introduces a spin $S$ by defining the canonical momentum $P$ as

$P=\mu v-s \times r i r^{2}$

where

$s \cdot r / r=-\left(a_{1} a_{m 2}-a_{2} a_{m 1}\right) / c$

Wit: this definition of $S$, Eq.(66) yields the familiar relation $J=r \times p+s$ $=L+S$. The properties of $P$ and $S$ are indeed those suggested by this familiar combination involving the croital angular momentum vector $L$. Thus, one sees that the electromagnetic angular momentum of Eq.(66), which is a consequence or the velocity dependent interaction between the particles, plays the role of a spin angular momentum. Here, we have used the fact that $g(r / r) / d t=(v / r)-r(r \cdot v) / r^{3}=r \times v \times r / r^{3}$. Schwinger then goes on tc construct a picture in which hadronic matter is viewed as a magnetically neutral 
composite of dual-charged particles or dyons that are based electrically on a new unit of charge $q_{0}=q / 3$ (i.e., one-third the usual electronic charge), and $a_{m o}=a_{m} / 3$.

For the Landau cyclotron motion

$$
\begin{aligned}
P & =\mu v+q A / c=\mu v+(q / 2 c) B \times r=\mu v+(q / 2 \pi c)\left(\pi r^{2} B\right) \times r / r^{2} \\
& =\mu v+(q \Phi / 2 \pi c) i_{z} \times r / r^{2}=\mu v-5 \times r / r^{2},
\end{aligned}
$$

where, in analogy with Eq.(68), the spin operator for this problem is $S=$ $-(q \Phi / 2 \pi c) i_{z}$, and $\Phi$ is the magnetic flux. Then, $r \times p=r \times \mu v-r \times s \times r / r^{2}=$ $r \times \mu v-s(r \cdot r) / r^{2}+r(r \cdot s) / r^{2}$. That is the total angular momentum,

$$
J=r \times p+S=L+S=r \times \mu v+r(r \cdot S) / r^{2}=L_{F}+r(r \cdot S) / r^{2} .(71)
$$

Equation (70) for the Landau cyclotron motion is equivalent to Eq.(68) of Schwinger's charge-monopole composite. Here again, $P$ and $S$ of Eqs.(70) and (71) are those suggested by the familiar combination involving the orbital angular momentum vector $L$. Thus we see that the wilczek's flux factor $\left(-q \Phi i_{z} / 2 \pi c\right)$ plays the role of the spin operator $s$ for the charged particle-flux tube composites, just the same way as schwinger's $S$ defined by Eqs.(68) and (69) plays the role of spin for the charge-monopole composites or dyors. We note that $[r \times(p-\mu v)]=(q / c) r \times A=(q B / 2 c) r \times i_{z} \times r=(q B / 2 c)\left[r^{2} i_{z}\right.$ $\left.-r\left(r \cdot i_{z}\right)\right]=(q \Phi / 2 \pi c) i_{z}-(q B / 2 c) r\left(r \cdot i_{z}\right)=-S-(q / 2 c) r(r \cdot B)=-S-(q / 2 c)$ $r(r \cdot \nabla \times A)$. That is, for this Landau cyclotron motion, we have

$$
S=-r x(p-\mu v)-(1 / 2) r[r \cdot \nabla x(p-\mu v)] \text {. }
$$


where $(p-\mu v)=q A / c$. Also from Eqs.(68) or (70), we get

$S=-r \times(p-\mu v)-r(r \cdot s) / r^{2}$.

We pointed out earlier that when the generalized potential $U$ of any physical problem is velocity dependent, $D \neq \mu \mathrm{v}$. In this case, following Schwinger, we can define a generalized spin operator $S$ for such problems with the aid of Eqs.(72) and / or (73). Such a definition of spin might prove useful in examining the connection between the spin and statistics of composite particles, such as for example, the charge-monopole composites discussed by Goldhaber. 25 charged particle-flux tube composites discussed by wilczek 10 Here "statistics" is used, in the sense of Goldhaber, to mean "permutaticr. symmetry of indistinguishable-particle wave functions." Further, if this spin $S$ takes integral values, then the total angular momentum $J$ is integral, and the problem will have an exact schrodinger's spatial representation. However, if $S$ is half-integral, then the problem under study has no exact spatial representation. For the Landau gyration states, $s=-(q \Phi / 2 \pi c) i_{z}=-i_{z}(n$ 1/2)h, using the $\Phi_{n}$ of Eqs.(10) or (57). Thus, we see that these Landau gyration states do not have an exact Schrodinger's spatial representation.

We wish to conclude this section by pointing out some of the difficulties that one may encounter in the spin-statistics connection of physical problems which have velocity-dependent generalized potentials $U$. First and foremost, cre does nct know a priori that this problem indeed has an exact jchrcdinger's spatial representation. Only in this representation, we have $P=-i \pi \nabla$, and $L$ $=-\operatorname{Mr} \times \nabla$, and the ordinary spherical harmonics are the eigenfunctions of the croital arigular momentum cperator. As Goldhaber25 states. "The usual correctior between spir and statistics allowed for point particles ir iccal 
field theory is equivalent to the requirement that a pair of distinguishable particles have an even $L+S$, where $L$ is the relative orbital angular momentum and $S$ is the total spin of the pair. This may be reduced to the common statement about symmetry or antisymmetry of wavefunctions under interchange of particle coordinates, provided certain phase conventions are established. In particular, one depends on the choice of angular momentum eigenfunctions which acquire a phase $(-1)^{L}$ upon inversion of relative position coordinates $r$. Ordinary spherical harmonics exhibit this behavior. They are eigenfunctions of the orbital angular momentum operator - if $r \times \nabla$, meaning that the relative momentum operator is $-i \hbar \nabla$. However, magnetic effects imply the existence of velocity-dependent potentials which may (and in this case do) alter the phase produced by the inversion of $r$." For our case, for example, it appears that in the cylindrically symmetric gauge, the Landau cyclotron motion about its guiding center cannot have an exact schrodinger's spatial representation. This is apparent from the fact that the $z$-component of its canonical angular momentum takes on half-odd-integral values, the g-value is two and not one, and has a zero-point gyration state whose behavior is analogous to that of the zitterbewegung of the Dirac's free particle so that spin $1 / 2$ is its orbital angular momentum. 16.15 However, Landau 13 has shown that this problem has a Schrodinger's spatial representation in the cylindrically nonsymmetric gauge. In this representation, the eigenfunctions are the Hermite polynomials, and the motion is a one-dimensional harmonic motion instead cf the actual circular harmonic motion. Hence, in this representation it is not clear whether one can choose the ordinary spherical harmonics, which are the orbital angular momentum eigenfunctions, as also the eigenfunctions for energy with the correct Landau energy eigenvalues. Alternatively, it would appear that scme linear combination of the Hermite polynomials in $x$ and $y$ cocrdinates with a phase difference of $\pi / 2$ may play an important role ir the study cf motions whose total orbital angular momentum takes nalf-odd-integral values of $x$. 
VIII. SUMMARY AND CONCLUSIONS

We have presented a rather critical but theoretically well-founded comprehensive analysis of the angular momentum, magnetic moment, g-value, and the magnetic flux associated with the Landau gyration states. This Landau problem is a particular case of a more general class of problems where the generalized potential $U$ or their Lagrangian is velocity dependent. For such problems, the canonical momentum $P$ is not equal to the mechanical momentum $\mu \mathrm{v}$. In the literature, there is considerable confusion as to how one should define the orbital angular momentum $L$ of such systems. Is it $r \times p$ or is it rxuv?. In general, in quantum mechanics, do these problems with the velocity-dependent $U$ have an exact schrodinger's spatial representation always? What is the g-value of such problems?. Is it the classical value of one, or is it like spin with the value two, or is it something else 33 ?. In this paper we have presented theoretically well-founded answers to these and other somewhat related problems. These answers may appear very surprising, just as many physicists were rather shocked when Bohm and Aharonov first brought up the issue that the vector potential $A$ is a "real" field that is a measurable quantity. Indeed, all the results discussed here are in some sense connected to this Aharonov-Bohm effect. As Feynman says, ${ }^{2}$ it is interesting that something like this can be around for many years but, because of certain prejudices of what is and is not significant, continues to be ignored. We also find that the possibility exists that the experimentally observed fundamental unit of flux quantum as $\mathrm{hc} / 2 \mathrm{q}$ instead of as $\mathrm{hc} / \mathrm{q}$ need not necessarily imply the existence of "electron pairs" of the BCS theory of superconductivity, a rather controversial, speculative, and outrageous result. Finally, we find that, for velocity-dependent generalized potential $U$, the particle-field interacticr contribution to the total angular momentum plays the role of a spir angular momentum $S$ which is determined by the departure $(p-\mu v)$, in agreement with schwinger's dyon theory of hadrons. According to Goldhaber and also 
Wilczek, this spin will determine the statistics of the system.

\section{ACKNOWLEDGMENTS}

This work was supported by the U.S. Department of Energy Contract No. DE-ACO2-76-CHO-3073. I thank Professor Arthur S. Wightman for his 1966-comments on some parts of this problem.

\section{REFERENCES}

1 H. Goldstein, Classical Mechanics (Addison-Wesley, Reading, Mass., 1957).

2 R.P. Feynman, R.B. Leighton, and M. Sands, The Feynman Lectures in Physics (Addison-Wesley, Reading, Mass., 1963), Vol.2.

3 F.S. Crawford, Am. J. Phys. 58, 337-344 (1990).

4 L.1. Schiff, Quantum Mechanics (McGraw-Hill, New York, 1955).

5 Claude Cohen-Tannoudji, Bernard Diu, and Frank Laloe, Quantum Mechanics (john Wiley \& Sons, Inc., New York, 1977) Vol.1.

$6 Y$. Aharcnov and D. Bonm. Phys. Rev. 115, 485-491 (1959).

7 J.D. Jackson, Classical Electrodynamics (John Wiley \& Sons, Inc., New York, 1975). 


\section{5}

8 W.K.H. Panofsky and M. Phillips, Classical Electricity and Magnetism (Addison-Wesley, Reading, Mass., 1956); J.M. Blatt and V.F. Weisskopf, Theoretical Nuclear Physics (John Wiley \& Sons, Inc., New York, 1952).

9 R.P. Feynman, Quantum Electrodynamics (W.A. Benjamin, Inc., New York, 1961).

$10 \mathrm{~F}$. Wilczek. Phys. Rev. Lett. 48,1144-1146 (1982).

11 F. Bloch, Phys. Today 19, No.5, 27-36 (May 1966).

12 H. Huang, Statistical Mechanics (John Wiley \& Sons, Inc., New York, 1963).

13 L.D. Landau and E.M. Lifshitz, Quantum Mechanics, non-relativistic Theory. Addison-Wesley, Inc., Reading, Mass., 1958).

14 P.A.M. Dirac, The Principles of Quantum Mechanics (Oxford U.P., Oxford, England, 1957).

15 V. Arunasalam, Am. J. Phys. 38, 1010-1022 (1970).

16 A.O. Barut and Nino Zanghi, Phys. Rev. Lett., 52, 2009-2012 (1984).

17 P.A.M. Dirac, Phys. Today, 23, No.4, 29-31 (April 1970).

18 E.S. Deaver and W.M. Fairbank. Phys. Rev. Lett. 7, 43-46 (1961); R. Doll and M. Nabauer, Phys.Rev. Lett. 7, 51-52 (1961).

19 J. Eardeer, L.N. Cooper, and J.R. Schrieffer, Phys. Rev. 108, 1175-1204 $(1957)$. 
20 Lars Onsager, Phys. Rev. Lett. 7,50 (1961).

21 N. Byers and C.N. Yang. Phys. Rev. Lett. 7, 46-49 (1961).

22 Julian Schwinger, Science 165, No.3895, 757-761 (22 August 1969).

23 J. Schwinger. Phys. Rev. 144, 1087-1093 (1966); ibid. 173, 1536-1544 (1968): D. Zwanziger, Phys. Rev. 176, 1480-1488, and 1489-1495 (1968).

24 P.A.M. Dirac, Phys. Rev. 74, 817-830 (1948); A.S. Goldhaber and W.P. Trower, "Resource Letter MM-1: Magnetic monopoles", Am. J. Phys. 58, $429-439$ (1990).

25 A.S. Goldhaber, Phys. Rev. Lett. 36, 1122-1125 (1976).

$26 \vee$. Arunasalam. Phys. Essays 4, No.4 (December 1991).

$27 \mathrm{~V}$. Arunasalam, Princeton University Plasma Physics Laboratory Report, MATT-755, "On Half-Odd-integral Orbital Angular Momentum:" (February 1970), PP.8.

28 C. Kittel, Elementary statistical Physics (John wiley \& Sons, Inc., New York. (1958).

$29 \mathrm{~V}$. Arunasalam. Am. j. Phys. 37, 877-881 (1969): j. Schwinger, Phys. Rev. 76, 790-817 (1949); S.N. Gupta, Nature 163, 686-687 (1949): … Luttinger, Phys. Rev. 74, 893-898 (1948).

30 H.A. Bethe, Phys. Rev. 72, 339-341 (1947); ¿. Schwirger, Quantum Electrooynamics (Dover Publications, Inc., New York, 1958). 
3 i F. London, Phys. Rev. 74, 562-573 (1948); F. London, Superfluids (John wiley \& Sons, Inc., New York, 1950).

32 E. Witten, Phys. Lett.86B, 283-287 (1979).

33 c. Kittel, Phys. Rev. 76, 743-748 (1949). 
Dr. F. Paoloni, Univ. of Wollongong, AUSTRALIA

Prof. M.H. Brennan, Univ. of Sydney, AUSTRALIA Plasma Research Lab., Australian Nat. Univ., AUSTRALIA

Prof. IR. Jones, Flinders Univ, AUSTRALIA

Prof. F. Cap, Inst. for Theoretical Physics, AUSTRIA

Prot. M. Heindler, Institut für Theoretische Physik, AUSTRIA

Prof. M. Goossens, Astronomisch instituut. BELGIUM

Ecole Royale Militaire, Lab. de Phy. Plasmas, BELGIUM

Commission-European, DG. XII-Fusion Prog., BELGIUM

Prof. R. Bouciquie, Rijksuniversiteit Gent, BELGIUM

Dr. P.H. Sakanaka, Instituto Fisica, BRAZIL

Instituto De Pesquisas Espaciais-INPE, BRAZIL

Documents Ottice, Atomic Energy of Canada Lid., CANADA

Dr. M.P. Bachynski, MPB Technologies, Inc., CANADA

Dr. H.M. Skarsgard. Univ. of Saskatchewan, CANADA

Prof. J. Teichmann, Univ. of Montreal, CANADA

Prot. S.R. Sreonivasan, Univ. of Calgary, CANADA

Prof. T.W. Johnston, INRS-Energie, CANADA

Dr. R. Bolton, Centre canadien de fusion magnétique, CANADA

Dr. C.R. James., Univ. of Alberta, CANADA

Dr. P. Lukac, Komenskeho Universzita, CZECHOSLOVAKIA

The Librarian, Culham Laboratory. ENGLAND

Library, R61, Ruthertord Appleton Laboratory, ENGLAND

Mrs. S.A. Hutchinson, JET Library, ENGLAND

P. Măhönen, Univ. of Helsinki, FINLAND

C. Mouttet, Lab. Physique des Milieux lonisés. FRANCE

J. Radet, CEN/CADARACHE - Bat 506, FRANCE

Ms. C. Rinni, Univ. of loannina, GREECE

Dr. T. Mual, Academy Bibliographic Ser., HONG KONG

Preprnt Library, Hungarian Academy of Sci., HUNGARY

Dr. B. Das Gupta. Saha Inst. of Nuclear Physics, INDIA

Dr. P. Kaw, Inst. for Plasma Research, INDIA

Dr. P. Rosenau, Israel Inst. of Technology, ISRAEL

Libranian, Intemational Center for Theo Physics, ITALY

Miss C. De Palo, Associazione EURATOM-ENEA, ITALY

Dr. G. Grosso, Istituto di Fisica del Plasma, ITALY

Dr. H. Yamato. Toshiba Res \& Devel Center. JAPAN

Prot. I Kawakami. Atomic Energy Res. Inst., JAPAN

Prof. k. Nishikawa, Hiroshima Univ., JAPAN
Director, Japan Atomic Energy Research Inst., JAPAN

Prof. S. Itoh, Kyushu Univ., JAPAN

Data and Planning Center, Nagoya Univ, JAPAN

Prof. S. Tanaka, Kyoto Univ., JAPAN

Library, Kyoto Univ., JAPAN

Prof. N. Inove, Univ. of Tokyo, JAPAN

S. Mori, Technical Advisor, JAERI. JAPAN

O. Mitarai, Kumamoto Inst. of Technology, JAPAN

H. Jeong, Korea Advanced Energy Research Inst., KOREA

Prot. D.I. Choi, The Korea Adv. Inst. of Sai. \& Tech., KOREA

Prot. B.S. Liley, Univ. of Waikato. NEW ZEALAND

Inst. of Plasma Physics, PEOPLE'S REPUBLIC OF CHINA

Librarian, Inst. of Physics, PEOPLE'S REPUBLIC OF CHINA

Library, Tsinghua Univ., PEOPLE'S REPUBLIC OF CHINA

Z. L. S.W. Inst Physias, PEOPLE'S REPUBLIC OF CHINA

Prof. J.A.C. Cabral, Instituto Superior Tecnico, PORTUGAL

Dr. O. Petous, AL I CUZA Univ., ROMANia

Dr. J. de Villiers, Fusion Studies, AEC, S. AFRICA

Prof. M.A. Hellberg, Univ. of Natal, S. AFRICA

C.I.E.M.A.T. Fusion Division Library, SPAIN

Dr. L. Stenflo, Univ. of UMEA, SWEDEN

Library, Royal Inst. of Technology, SWEDEN

Prof. H. Wilhelmson, Chalmers Univ. of Tech., SWEDEN

Centre Phys. Des Plasmas, Ecole Polytech, SWITZERLAND

Bibliotheok, Inst. Voor Plasma-Fysica. THE NETHERLANDS

M. Durgut. Vico Chairman. Middle East Tech. Univ., TURKEY

Dr. D.D. Ryutov, Siberian Branch of Academy of Sci., USSR

Dr. G.A. Eliseov, Kurchatov Inst., USSR

Librarian, The Ukr.SSR Academy of Sciences, USSR

Dr. L.M. Kovrizhnykh, Inst. of General Physics, USSR

Kemforschungsanlage GmbH. Zentralbibliothek, W. GERMANY

Bibliothek, Inst. Für Plasmaforschung, W. GERMANY

Prof. K. Schindler, Ruhr-Universitat Bochum, W. GERMANY

Dr. F. Wagner, (ASDEX), Max-Planck-Institut, W. GERMANY

Librarian, Max-Planck-Instutut, W. GERMANY

Prof. R.K. Janev, Inst. of Physics, YUGOSLAVIA 

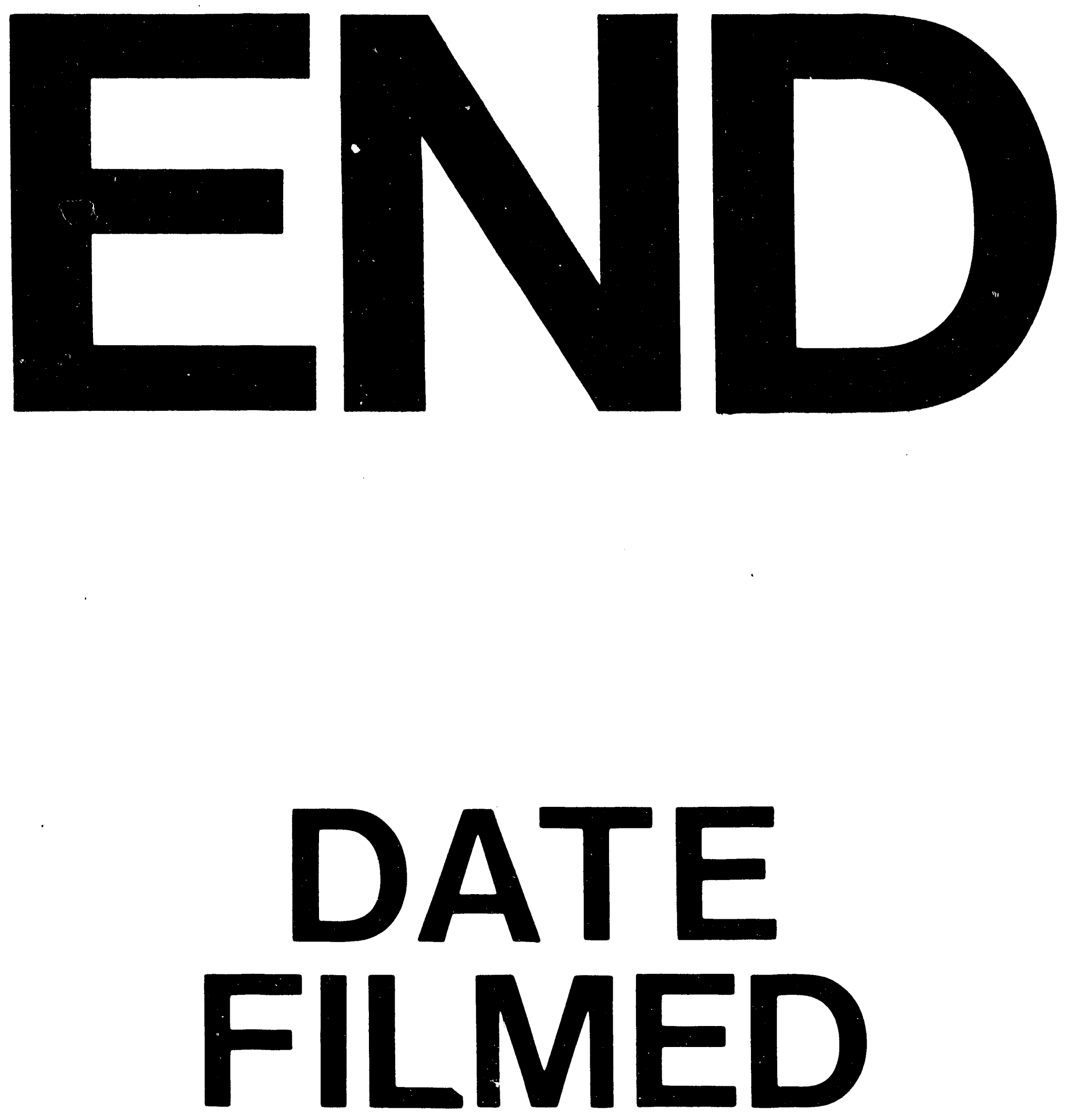

i

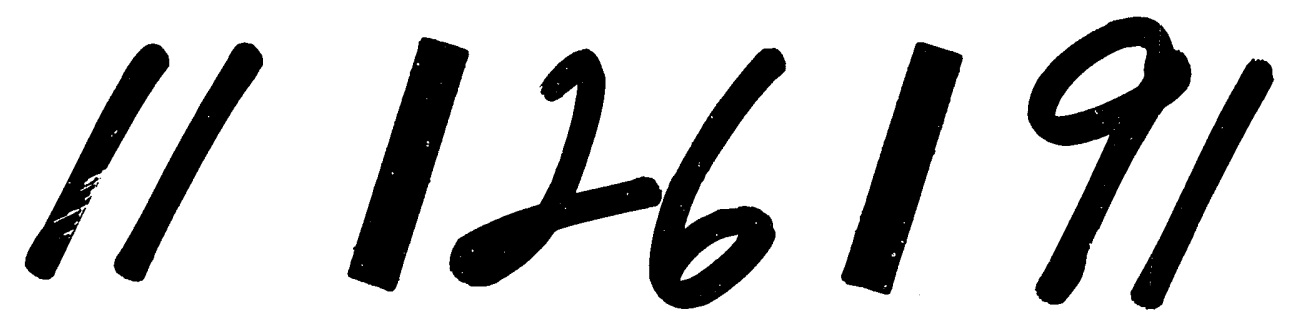

- 


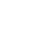

